\title{
Contribution à l'étude du sacrifice chez les Minyanka
}

Contribution to the study of sacrifice among the Minyanka

Danielle Jonckers

\section{(2) OpenEdition}

1 Journals

Édition électronique

URL : http://journals.openedition.org/span/314

DOI : 10.4000/span.314

ISSN : 2268-1558

Éditeur

École pratique des hautes études. Sciences humaines

\section{Édition imprimée}

Date de publication : 1 septembre 1976

Pagination : $91-110$

ISSN : 0294-7080

\section{Référence électronique}

Danielle Jonckers, « Contribution à l'étude du sacrifice chez les Minyanka », Systèmes de pensée en Afrique noire [En ligne], 2 | 1976, mis en ligne le 04 juin 2013, consulté le 30 avril 2019. URL : http:// journals.openedition.org/span/314 ; DOI : 10.4000/span.314 


\author{
C O N T R I B U T I O N A L 'É T U D E \\ DU SACRIFICE CHEZ LE S M I N A N K A \\ par \\ Danielle Jonckers
}

\title{
I. Introduction.
}

L'unitê formelle du fait sacrificiel est trompeuse. En tant que résultat factuel, le sacrifice masque une diversitê de processus qu'il est impossible de réduire à un objet d'étude unique et homogène.

Les deux niveaux principaux d'analyse sont l'étude du sacrifice en tant que partie d'un système symbolique d'une part, et d'un système de relations sociales d'autre part. Le présent article a pour but de poser, pour le cas minyanka, les jalons de ces deux démarches. En ce qui concerne la première, une prise de position s'impose : doit-on faire "le tableau de la thêorie ou la théorie de la réalité indigène" ? (1). Lévi-Strauss a pu reprocher à Mauss d'avoir fait appel, pour expliquer la magie et le don, à des notions métaphysiques empruntées à une théorie indigène. D'autres ont tenté d'introduire, dans les systèmes religieux, de mystérieuses sources d'énergie qui n'ont même plus le mérite de correspondre à des catégories indigènes. La complexité des phénomènes religieux en gênêral et du sacrifice minyanka en particulier commande que 1 'on observe, quant à leur explication, une certaine prudence et que 1 'on dresse d'abord l'inventaire des catêgories indigènes. C'est d'ailleurs Mauss lui-même qui disait :

"Étudier une société quelconque (...) ne permettra jamais à personne de

(1) Selon 1'expression de $\mathrm{C} 1$. Lévi-Strauss, Introduction à 1'oeuvre de Mauss, préface à Sociologie et anthropologie de M. Mauss (Paris, P.U.F., 1950), p. xxxix. 
conclure d'une façon générale sur la mentalité humaine ; mais la découverte de nouveaux concepts, de nouvelles catégories, n'en constitue pas moins un apport précieux pour l'histoire de la pensée humaine." (2).

II. Le sacrifice dans le système religieux minyanka.

La pensée religieuse minyanka intègre tous les éléments de l'univers, toutes les manifestations naturelles et sociales; elle lie les hommes entre eux et.leur garantit une articulation harmonieuse avec leur environnement. Les rituels marquent tous les actes de la vie; ils orientent les forces naturelles et aident les homes dans leurs entreprises.

Malgrê 1'existence, dans presque tous le villages, d'une communautê musulmane de plus en plus nombreuse, les cultes traditionnels imprègnent encore totalement la vie de ceux qui les pratiquent; ils n'ont cessé d'être facteurs de cohésion et garants de l'ordre social. La sociêté minyanka tente de domestiquer, par le sacrifice, toutes les violences qui 1'agitent ou la menacent (la mort, la maladie, la guerre, la sécheresse) et affirme ainsi ses prétentions à la durée.

L'enquête n'a pas révélé, comme chez les Dogon par exemple, le thème $d$ 'un héros mythique dont la mise à mort serait perpêtuellement. réactualisée par les rites sacrificiels. Ceux-ci occupent néanmoins, dans les pratiques religieuses minyanka, une place centrale.

Les sacrifices collectifs ont lieu à deux moments de l'année : après les récoltes en novembre-décembre, et avant l'hivernage, en marsavril. Les sacrifices individuels sont occasionnels ou prennent place après les précédents, lors des cérémonies semestrielles.

Les institutions religieuses minyanka se divisent en deux grandes catêgories : les cultes gênêraux et les sociétés d'initiation (3). Les premiers comprennent les cultes rendus :

-- au Dieu suprême, klè,

-- aux ancêtres,

(2) Marcel MauSS, Manuel d'ethnographie (Paris, Payot, 1967), p. 205.

(3) Voir J.-P. COLlEYN, Notes sur la pensée religieuse des Minyanka, Systèmes do pensée en Afrique noire, cahier 1975 (Paris, L.A. 221, 1975), pp. 19-34. 


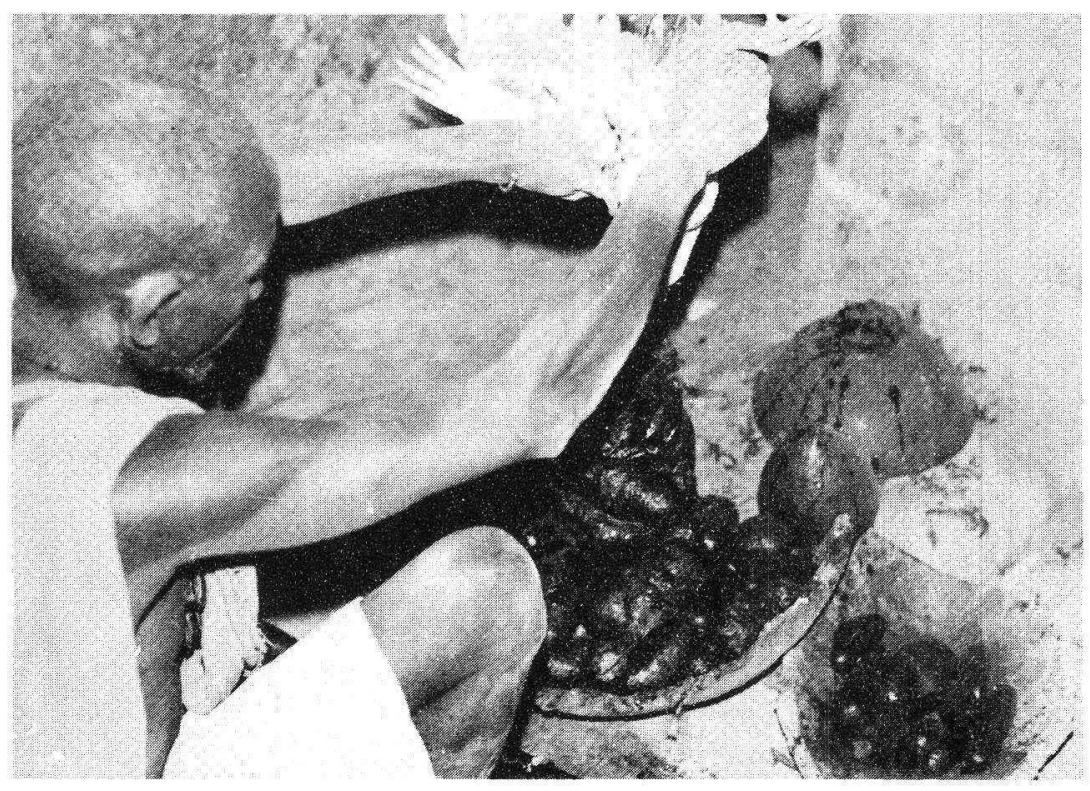

Planche I. Sacrifice sanglant sur les autels portatifs du ka Chyaka (chose puissante, gros sac), yapèrè qui rassemble entre autres les éléments du nya et du nankon. Village de Wolosso. 
-- à l'enciume (tumpugno), censée être le plus vieux principe sacré du village, donnée aux hommes par le vautour mythique,

-- au kanchys ("jarre du village"), autel de fondation du village,

-- à tyelé ("1a vieille femme"), première femme mythique,

- au kachikan, bois sacré abritant les forces associées à la terre,

- au dasimi, protecteur du village, chef de village au plan symbolique,

-- à fari, génie de l'eau.

Dans ces cultes, le sacrifice apparaît surtout comme un rite cyclique obligatoire. Les autels fixes sur lesquels il s'effectue sont censés être aussi vieux que le village ou même lui avoir préexisté; ils échappent aux vicissitudes humaines. Il n'existe pas de termes génériques pour désigner ces autels; ils portent en général le nom de l'entité religieuse à laquelle ils se rapportent. Ils sont souvent constitués d'une jarre (parfois recouverte de pisê) contenant des fragments de l'environnement naturel, des éléments évoquant les principales figures mythiques et des reliques des ancêtres. Parmi ces cultes, celui rendu à klè occupe une place particulière. L'Être suprême reçoit régulièrement des libations de bière, de crème de mil et de sang de poulet. Il existe à cet effet, dans tous les villages, des autels klè, qui se présentent sous la forme de cônes en pisé d'une soixantaine de centimètres de haut. klè est par ailleurs invoqué dans toutes les prières qui précèdent les sacrifices, qu'il s'agisse des cultes généraux, du culte des ancêtres ou des activités rituelles des sociétés initiatiques. Cependant, et c'est là le point important, les sacrifices ne sont pas indispensables à l'existence même de klè : Dieu est une figure lointaine, distante des hommes et du monde matériel.

Les sociétés d'initiation dominent la vie religieuse des Minyanka. Les plus importantes sont :

-- le nya ( ou wara), protecteur du village contre les sorciers; associè à la fêconditê,

-- 1e sandogno ("hyène", monitrice de la culture),

-- le nankon, institution religieuse des chasseurs, la plus puissante de toutes les sociétés d'initiation,

-- le manyan, culte réputé le plus ancien, associé à la guerre. Il se caractērise par la taille imposante de son matériel rituel. 
Ces institutions comportent :

-- les sociétaires ; les maîtres du culte (yapèrèfo), les possédés (tutumo), les bouffons (korduba), les musiciens (tyeli), le sacrificateur et les initiés,

-- un sanctuaire,

-- les yapèrè, c'est-à-dire les autels portatifs qui appellent les sacrifices sanglants.

Les sociétés d'initiation se veulent dépositaires de toutes les valeurs morales, qui sont elles-mêmes en relation avec les principes cosmiques. Le culte s'adresse tout d'abord à Dieu, mais aussi à des entités religieuses (nya, nankon, manyan...), placées elles-mêmes sous le patronage de hêros mythiques. Ces hêros mythiques, qui sont soit des créatures fantastiques, soit des personnages d'apparence humaine ou animale, ont donnê aux hommes une partie de la connaissance du monde. Ils leur ont enseigné les moyens d'agir, grâce à cette connaissance, sur le système cosmique, par la fabrication d'autels (yopèrè) et 1 'exécution de rites appropriés dont le plus important est le sacrifice.

La notion de yapéré est complexe. La conjonction des principaux yapèrè réalise un véritable microcosme (4). Le yapèrè est un objet fabriqué (ya signifie chose fabriquée), un amalgame de fragments divers chargés de forces dangereuses, de nyoma. Dans les chants religieux du nankon on dit :

"prends tes bagages, tes bagages de sorcellerie, prends tes bagages, tes bagages de nyama."

Le terme yapèrè est intraduisible en français. Son étymologie n'a d'ailleurs pu être établie avec certitude. La traduction par "autel portatif" est sans doute la plus neutre mais elle est imparfaite : elle ne rend pas compte de 1 'incorporation de l'entité religieuse dans l'objet ni de son caractère redoutable, que soulignent par exemple ces louanges :

(4) Comme le montre Ph. JESPERS, Contribution à l'étude des autels sacrificiels chez les Minyanka, Systemes de pensée en Afrique noire, cahier 1976 (Paris, L.A. 221, 1976). 
"épervier, vieux chien (hargneux), cette voix ressemble à celle du lion ce tas de choses ressemble au lion véritê, le lion arrête le lion (sorcier) dans la brousse quelle est la chose qui a le nom de l'éléphant c'est le nankon qui est le plus redoutable redoutable, redoutable, dasiri (5) des hommes ton nom est connu partout nankon."

"que toute personne qui trahit et qui est venue ici que le manyan la tue qu'il lui arrache le foie et le coeur manyan voici ton couteau pour percer ton ennemi

si tu trouves une sorcière perce lui le fond et prends lui le foie et le coeur."

"nankon, sorcier des yapère (yapere sikanfolo)

les autres yapèrè n'ont qu'à te laisser la sorcellerie."

Le yaperè n'est pas simple réceptacle : ses éléments constitutifs sont à la fois esprit et matière. L'existence et la puissance du yapèrè dépendent du rite sacrificiel : le sang augmente son énergie vitale. C'est d'ailleurs le sang coagulê des nombreux sacrifices qu'il reçoit qui donne au yapèrè son aspect goudronné. L'épaisseur de cette croûte brunâtre révèle l'âge du yapèrè et la puissance de son énergie contenue. Les yapèrè sont en quelque sorte des "outils spirituels" ; ils sont destinés à agir sur le modèle des entités religieuses. La fabrication de ces objets institue une certaine autonomie magique de 1 'homme par rapport au système du monde. Quoique dangereux et chargểs de nyama, les yapèrè n'ont de sens que dans leur subordination (même imparfaite) à l'homme. Le yapèrè est inclus dans l'ordre cosmique (qu'il rësume souvent) mais moyennant certaines précautions et par 1'intermédiaire du sacrifice, 1 'homme peut à tout moment le soumettre à ses propres fins. Cette intervention de 1'homme, dans 1'ordre de 1'univers, grâce à la médiation des yopèrè souligne a contrariola nature spécifique de l'Être suprême $k Z \grave{e}$ en tant que pur esprit.

(5) Cette expression bambara signifie littéralement "attacher la bouche ; elle s'emploie pour désigner toute pratique magique destinée à prendre le contrôle sur quelqu'un. A ne pas confondre avec le dasiri, protecteur du village qui "attache la bouche" des ennemis. 
III. Les categories sacrificielles.

Le substantif kara, formé à partir du verbe $k a$ (donner), est le terme générique recouvrant 1 'ensemble des offrandes, sanglantes ou non, qu'elles soient collectives et cycliques, ou individuelles et occasionnelles. Les mots kara ou kachin sont utilisês indifféremment à Koutiala et dans l'est du Minyankala, mais dans le nord kachin s'applique à l'ensemble d'un rituel et kara au moment précis de la mise à mort d'un animal. Les animaux les plus couramment exécutés sont les poulets, les chèvres, les moutons, les chiens et plus rarement les boeufs. Signalons une particularité du nankon (yapèrè patronné par les chasseurs); il est le seul à recevoir en plus du sang des animaux domestiques, celui d'animaux sauvages capturés vivants et plus spécialement celui de 1 'hyène, de 1 'oryctéropte, du boa et de l'antilope. L'installation d'un nankon dans un village commence par une chasse sacrificielle. Tout chasseur possède un "anneau du nankon" (nankonkapara), autel portatif sur lequel il achève les animaux blessés à la chasse. La viande sera partagée et consommée par tous les initiês. Si le chasseur tue le gibier sans verser son sang sur l'autel, il est libre d'en disposer.

Certains termes soulignent la finalitê des sacrifices individuels : .

(a) nyèrègè est un substantif dêrivê du verbe nyèrè (solliciter); il désigne le sacrifice le plus courant par lequel les homes demandent à $k$ lè, aux ancêtres, aux yapèrè, de leur accorder des bienfaits (santê, prospérité, féconditê); adressêe à certains yapèrè, la demande peut consister en une visée agressive, proche de la sorcellerie. Le sacrifiant énonce sa demande et le sacrificateur la répète :

"Il te donne cela grâce à Dieu

il donne au nya de jour comme de nuit

tout ce qu'il cherche dans le monde cherche le et donne lui de jour et de nuit

tout le bonheur qu'il cherche, cherche le et donne lui de jour et de nuit."

"Toi, nya, donne la santē à ses enfants, donne lui le succès." "C'est la vérité, manyan, cette femme est venue avec ce poulet pour te le donner

garde ses enfants en bonne santê et fais leur un bon hivernage fais cesser les querelles qu'il y a entre les enfants de cette femme toute parole de querelle, laisse cela de nuit et de jour et prends ce sacrifice." 
(b) nyèrègè s'accompagne le plus souvent de la promesse nyofaga ( $f a l a)$ (bouche échanger) d'effectuer un autre sacrifice si la demande est exaucée, celui-ci est appelé nyo faga wozo (bouche échanger retirer ou délivrer). Exemple :

"I1 dit qu'il a fait un voeu sur toi manyan

si tu vois qu'il vient donner ce qu'il a promis

c'est que le voeu a été réalisé

voici les poulets promis, prends les de nuit comme de jour."

(c) Les sacrifices ponctuent et favorisent le déroulement harmonieux de la vie mais le désordre est toujours possible et doit être compensé par des sacrifices appropriés. Le ka ya na (chose, réparer, a) est un sacrifice offert pour effacer les fautes commises consciemment ou inconsciemment. C'est le yapèrètutumo (envoyé du yapèrè), un médium, qui révèle ces ruptures d'interdits involontaires. Les fautes volontaires graves (adultère, sorcellerie,...) sont sanctionnées directement par le yapèrè lui-même, par la mort. Avant d'ensevelir les coupables, il faut effectuer des sacrifices yahnga (ce substantif est formê à partir du verbe yah, "1aisser").

Quelle que soit leur finalité, les sacrifices se déroulent selon le même rituel. Seuls les incantations et le choix de la couleur des victimes précisent leur spécificité. Lors d'un sacrifice à klè, on ne peut utiliser que des poulets blancs, signe de pureté, de sécurité. C'est la raison pour laquelle les yapèrè qui trempent dans la sorcellerie, pour mieux lutter contre elle, refusent les poulets blancs. On tuera un chien noir sur le nya pour éliminer un sorcier. Sur les autels de sorcellerie on immole des poulets rouges ou noirs, couleurs symbolisant la violence, le mal, la mort. La couleur joue un rôle important dans les prescriptions des devins; à défaut du poulet requis, on offre tout objet (cola, tissu, etc.) de la même couleur.

Les sacrifices collectifs cycliques rassemblent les diffërents objectifs des sacrifices individuels. Pendant l'oblation, le yapèrè fo ("celui qui a" le yapèrè), le chef de famille ou de village, demande le pardon des fautes commises, remercie pour les bienfaits obtenus et sollicite les puissances religieuses :

"Toutes paroles que $j$ 'aurais dites ou que quelqu'un qui me suit (cadet) ou qu'une femme aurait dites, voici leur eau (de pardon), si je t'offense pardonne cette offense nya."

"Merci pour tes actes, c'est pour cela que tu as été choisi." 


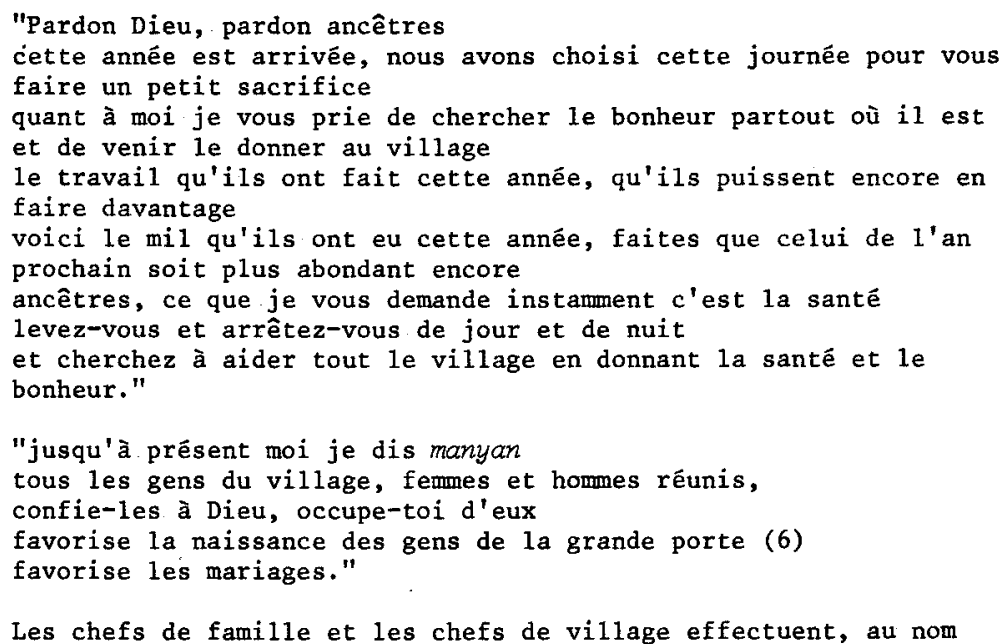
de la collectivité qu'ils représentent, les sacrifices sur les yapèrè familiaux et sur les autels consacrés à klè, aux cultes généraux ou aux ancêtres. Les sacrifices aux yapèrè exigent l'intervention d'un sacrificateur qui porte une tenue spéciale (pagne blanc) ou un insigne (ceinture) qui le distingue des sacrifiants et des autres initiés. La veille ou les jours qui précèdent la cérémonie, il ne peut avoir de rapports avec une femme et doit se laver avant et après les opérations sacrificielles.

Tous les sacrifices évoqués jusqu'à présent relèvent de la catégorie kara. D'autres types d'offrandes peuvent être isolés ; elles se dëroulent sans sacrificateur, elles ne nécessitent ni lieu de culte, ni autel.

Zoé wo (eau, verser) est un ka ya na (voir page précêdente) qui se fait pour une faute bénigne, telle que l'infraction à un interdit alimentaire ou un blasphème. Le rite consiste à verser un peu d'eau sur le sol.

Le saraga (mot d'origine arabe) consiste en général en une offrande de cola, de cendres, de coton, de cauris, déposée à un carrefour; mais le sacrifiant peut aussi abandonner un poulet ou une chèvre en

(6) Chaque concession lignagère est entourée d'une enceinte qui 8 'ouvre sur une porte monumentale, lieu de sacrifice aux ancêtres. 
brousse. Il s'adresse à klè, aux ancêtres, ou à toute entité religieuse. Il s'exécute la nuit ou à l'aube; le sacrifiant est seul et n'attend aucun signe d'acceptation ou de refus de son geste. Le saraga s'accomplit après consultation du devin ou suivant 1 a recommandation d'un yapèrè tutumo; ceux-ci peuvent parfois conseiller un sacrifice à un yapèrè.

Sous l'influence de l'islam, le terme saraga désigne de plus en plus fréquemment tout sacrifice. Le saraga au sens strict se fait essentiellement pour conjurer une maladie, un mauvais sort. Mais il sert aussi à lancer un maléfice. Il a lieu alors au crépuscule. Tandis que par exemple on brûle un mélange de poudre à fusil, de piments, de poils (de chien, de singe, de chat, de souris), en dirigeant la fumée vers ceux à qui on veut nuire, on récite l'incantation suivante :

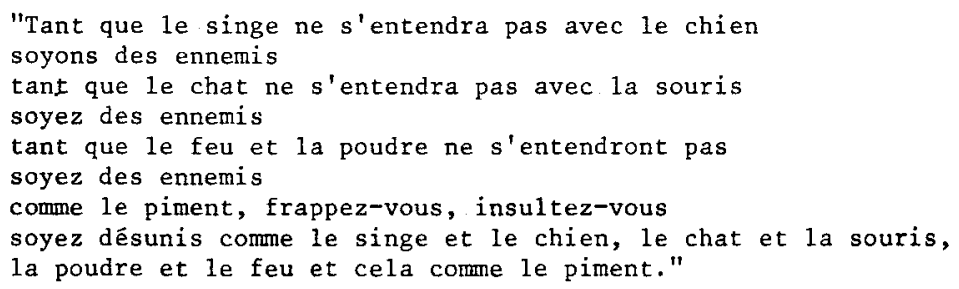

IV. La séquence sacrificielze.

Les cérémonies religieuses commencent soit le jeudi soir, pour se terminer le vendredi soir, soit le dimanche pour prendre fin le lundi. Les kara (voir supra), qui intêressent la collectivité, s'effectuent en public, sur les autels fixes et portatifs. Pour que tous soient concernés, approuvent et participent aux sacrifices, les victimes et le couteau sont présentés à l'assemblêe; ensuite, chaque offrande circule de main en main parmi les notables selon un ordre fixé par l'envoyé du yapèrè ou simplement déterminê par l'âge. L'immolation des victimes est toujours précédée d'une offrande d'eau pure et de mil, sous forme de crème ou de bière, qui fait partie intégrante du sacrifice. Pour les ancêtres et certains yapèrè, on verse de la cendre avant l'eau et la bière. La cendre est associée à I'idée de pureté, l'eau à la fécondité, le mil à la prospérité. Le sacrifice est intégré au cycle vital : un rapport analogique s'établit entre celui-ci et la 
séquence eau-mil-sang ("sans eau on n'a pas de mil, et sans mil il n'y a pas de vie, ni animale (poulets) ni humaine.") :

"Grâce à Dieu, grâce à tes anciens possesseurs voici ta nouvelle année, voici ton eau manyan prends-la de nuit comme de jour, favorise-nous la nuit et le jour de ta nouvelle année."

"Nous avons fixé le jour d'aujourd'hui pour ton sacrifice voici l'eau, voici l'eau dont je verse ta part je la crache sur toi pour rafraîchir ton corps voici l'eau de la crème de mil que tu nous as donné, avec la santé voici l'eau de mil

voici l'eau de la bière de mil que tu nous as aussi donné

le sacrifice va commencer maintenant venez, nous allons sacrifier au nya."

La cendre, l'eau et la bière, ou la crème, sont versés sur le sol par le yapère fo, ou le sacrificateur, à l'entrée du sanctuaire. Ces libations sont suivies d'un premier sacrifice de poulet qui a lieu au même endroit. Ensuite, l'envoyé du yapèrè, en état de transe, sort les autels portatifs du sanctuaire et les porte sur le dos (ou les traine), accompagné par les musiciens et la foule, à la périphérie du village, dans l'enclos où se dérouleront les sacrifices. Après avoir été imbibês de sang sacrificiel, les yapèrè sont ramenés au village par le possédé qui subit une seconde transe. Avant de pểétrer dans le sanctuaire, les objets sacrés reçoivent un dernier sacrifice, devant l'entrée. Ces deux processions fournissent à tous les participants 1'occasion de faire un don au yapèrè, c'est-à-dire au yopèrè tutumo. Ces offrandes sont des nyo faga wolo et des saraga (voir supra). Seuls les initiês et les personnes dont la naissance a été favorisée par le yapèrè peuvent pénétrer dans 1 'enclos sacrificiel. Les sacrifices qui y sont effectués concernent les différentes collectivités (village, confrérie religieuse, famille, société de culture). Ils sont suivis par les sacrifices individuels.

Le sacrifice ne peut être considéré ici comme un simple don ; il est l'opérateur principal d'un rite à caractère quasi-magique au cours duquel des forces vitales (nycoma) circulent, véhiculées par le sang, lequel ne peut en aucun cas toucher un non-initié. Le sang qui s'épanche sur les autels est traversê par deux courants : 1'un le vide de son nyama et l'autre le charge de celui du yapèrè. On balance les victimes au-dessus des autels pour les imprégner de cette nouvelle force. Tandis que le yopèrè est alimenté, que sa puissance est rénovée par le 100 
sang, la victime emmagasine le nyama du yapèrè. Les hommes qui la dépècent, la prêparent et la consomment assimilent une part de cette force. Le partage de la victime entre les initiés marque leur statut au sein de la société. Voici, par exemple, la répartition de la viande d'un chien sacrifié au nya : le dos revient au nyafolø, le gigot avant au nyatutumo, le cou à celui qui a chanté les louanges, la tête et les pieds aux équarisseurs, et le reste est distribué à tous les initiés. Le coeur et le foie sont écrasés et étalés sur les autels portatifs. Les victimes sacrificielles sont toujours consommées après avoir été vidées de leur sang sur les yapèrè. Il n'existe pas de terme particulier pour désigner ce repas rituel. La langue situe sur le même plan, apparemment profane, le repas des hommes et celui du yapèrè. On utilise pour désigner le premier l'expression nyagarağa (manger la viande du nya). Quant au possédé parlant au nom du yapèrè, il dira : "je suis venu manger à cet endroit. Le petit poulet, tuez-le; nous allons manger." Le yapèrè fo pourra également dire : "j"avais dit qu'en cette journée, le nya mangerait jusqu'à satiété et s'il est rassasié, il nous fera passer ce jour en bonne santé."

Autre remarque linguistique : on dira qu'on 'répare" (ya), qu'on "crêpit" (wologo) le yapere lorsque, après les sacrifices, on enduit soigneusement de sang ses différents éléments. Après l'égorgement, $1 \mathrm{e}$ sacrificateur ensanglante les autels portatifs et jette la dépouille de l'animal sur le sol. Les sacrifices de quadrupèdes sont toujours suivis de sacrifices de poulets. Pour ces derniers, chacun observe l'agonie et la commente, car les soubresauts indiquent si le sacrifice est accepté. Le poulet doit tomber une première fois sur le dos, ensuite se retourner deux fois et mourir sur le ventre; le sacrifice est refusé si le poulet tombe sur le ventre et s'immobilise. Dans ce cas, le sacrifiant consultera un devin, mais parfois le sacrificateur vérifie le verdict précédent au moyen de noix de cola. Après les avoir divisées en deux, $i 1$ les lance en implorant le pardon pour les fautes éventuellement commises dans 1 'exécution du rite. Il peut répéter l'opération trois fois. Si les cola retombent avec la face plate vers le haut, le sacrifice est enfin accepté. Le sacrificateur croque la noix, la mâche, crache une partie sur le yapère et consomme le reste. Toutes les puissances religieuses indiquent par certains signes si elles acceptent ou non le sacrifice. Ainsi par exemple, lors des sacrifices à la grande porte des ancêtres et à l'enclume, le poulet doit 
mourir sur le ventre pour être accepté et présenter des reins blancs après dépeçage. Si les reins sont noirs c'est qu'une faute a été commise et il faut compléter le sacrifice par des offrandes supplémentaires.

Toutes les incantations révèlent le souci des hommes de ne pas offenser même involontairement la puissance à laquelle ils s'adressent : ils s'inclinent avec beaucoup d'humilité devant des dieux et des ancêtres derrière lesquels se profile l'ordre social :

"Grâce à Dieu, grâce à tes anciens possesseurs, manyan, voici ta nouvelle année

tes anciens possesseurs ont dit que je te le donne je te le donne à leur manière moi je suis un enfant, je ne distingue pas ma droite de ma gauche je te donne à la façon de tes anciens possesseurs prends cela de jour comme de nuit je suis un enfant, je dis les paroles des vieux je suis l'envoyé des vieux."

\section{v. Les implications socio-économiques du sacrifice.}

Les critiques formulées par Marcel Mauss, Evans-Pritchard et Lévi-Strauss contre 1 'interprétation purement fonctionnaliste des phénomènes religieux sont trop connues pour que l'on y revienne. Mais s'il convient de réagir contre toute thêorie mécaniste (qu'elle attribue au fait religieux une fonction utilitaire ou qu'elle se borne à interpréter le rite comne simple reflet des relations sociales), il ne faut pas en conclure qu'il y a autonomie absolue du religieux par rapport aux autres instances du social. Si les faits religieux relèvent d'une organisation symbolique propre, ils s'inscrivent aussi dans la rêalité socio-économique. Tentons à présent le second type d'analyse en envisageant les pratiques religieuses comme partie intégrante des processus de production, de distribution et de consommation des biens matériels. Très souvent ce type d'analyse est négligé non seulement par les auteurs "culturalistes" mais aussi par les chercheurs trop strictement économisants. Ces derniers estimant que la connaissance travaille sur l'objet réel et non sur une matière dêjà transformée par eux, écartent de leurs préoccupations des sphères de la vie sociale qui chez nous ne s'inscrivent pas dans le système économique. Ainsi, la nature économique du sacrifice d'animaux domestiques à Dieu leur 
paraîtra-t-elle contestable sous prétexte que le destinataire est imaginaire. Si les trente chiens et la centaine de poulets offerts au nya de Wolobougou (au cours d'une des cérémonies semestrielles) 1 'avaient été non à une entité religieuse mais à un mâ̂tre de chair et d'os, l'implication économique de l'opération aurait été évidente. L'aspect économique des rites sacrificiels est loin d'être négligeable. En 1936, par exemple, soixante boeufs furent sacrifiés au kono (sociēté d'initiation d'origine bambara) du village de Debela. Actuellement, pour la plupart des cultes, dix à trente chiens (valant chacun de 30 à 50 francs français) et d'innombrables poulets (valant de 3 à 7 francs français) sont sacrifiés à chaque fête semestrielle. Le besoin de se procurer des animaux sacrificiels justifie la constitution de sociétés de culture qui se rendent en équipe (une vingtaine de jeunes honmes) sur les champs de villageois moyennant un chien ou une chèvre par journée de travail. Ces formes de coopération permettent de disposer à certains mơments précis du cycle agricole (dêfrichage, récoltes) d'une grande masse de main-d'oeuvre. A l'occasion des fêtes, des quantités énormes de bière de mil et de plats de riz sont préparés puis consommés. L'importance quantitative des biens produits puis dépensés à l'occasion des sacrifices nous renseigne sur les capacités techniques de la production. L'implication économique des activités rituelles ne repose pas ici sur le temps investi en cérémonies car (outre que la production elle-même n'est pas fondée sur l'économie du temps de travail) les rites se déroulent durant la saison sèche, période d'inactivité agricole. Le travail accompli par les femmes et des cadets en vue des activités rituelles a pour conséquence, à travers les sacrifices aux yapèrè et aux ancêtres, de confirmer l'autorité des aînés. La consommation des biens, fût-elle déterminêe par la religion, nous renvoie aux rapports sociaux de production. Ceux-ci règlent la distribution des produits dans la communauté familiale (7) et déterminent la nature des échanges avec l'extérieur.

La campagne minyanka est en pleine évolution, ce qui rend l'analyse difficile. Nous envisagerons successivement les implications socio-économiques des rites sacrificiels du temps de la préminence des communautés lignagères traditionnelles et depuis leur éclatement.

(7) D. JONCKERS et J.-P. COLLEYN, La communauté familiale chez les Minyanka, Joumal de la Soc. des Africanistes, XLIV, 1 (1974), pp. 43-52. 
A. Le mituel dans le cadre des communatés traditionnelzes.

Le chef de la communauté familiale (gbunfglg) est situé au pôle de la circulation des biens produits (8). A ce titre, il est le seul à pouvoir assumer les dépenses rituelles. Afin de faire face à ces obligations, 1'aîné sollicite de ses dépendants des prestations. Le travail sur les champs communs ( $f g r g b a$ ) débouche en partie sur la production du mil destiné aux repas cérémoniels et à la fabrication de la bière.

Il n'est pas exclu que les structures communautaires suscitent des processus visant à annuler les effets de facteurs susceptibles de modifier leur fondement même et que 1'oblation religieuse soit équivalente sur le plan écononique à la stérilisation d'une partie de la production. Les rites sacrificiels supposent une structure hiêrarchique qui commande le cycle prestation-oblation-redistribution. De cette redistribution sont d'ailleurs exclus les femmes et les non initiés. Les rites s'accompagnent, dans une atmosphère d'effervescence, d'une consommation importante de biens (surtout de viande et de bière). I1 peut sembler paradoxal que les principales fêtes religieuses se déroulent juste avant la difficile période de la soudure. On peut expliquer la fonction économique de ce "gaspillage" : il empêche la constitution d'un surplus et maintient donc un certain équilibre au niveau économique qui nie (à défaut de corriger) les inégalités dues aux facteurs naturels. La qualité variable des sols, les aléas démographiques, les dégâts des prédateurs constituent un milieu favorable à un enrichissement différentiel des familles. De plus et surtout, la brièveté de la saison des pluies, au cours de laquelle se concentrent les travaux agricoles, sanctionne durement les faiblesses humaines (maladie, paresse) qui ne sont pas toujours entièrement corrigées par les mécanismes de solidarité de la parenté.

La relation avec les "dieux" est d'abord réservêe aux chefs des communautés : 1'aîné parle au nom de ses dépendants. Dans la prière précédant le sacrifice au nya, par exemple, il dit : "toutes paroles que $j$ 'aurais dites ou que quelqu'un qui me suit (cadet) ou qu'une

(8) Ibid. note 7 
femme aurait dites, voici leur eau (de pardon)."

Les yapèrè ne sont pas seulement des choses matérielles ou des entités religieuses abstraites, ils sont, pourrait-on dire, "des rapports sociaux coagulés". Les vieux expliquent qu'un des devoirs des cadets et de femmes est d'accomplir les "travaux des yapèrè" (yapèrèmo-bara). Les jeunes hommes sont chargés de travailler en sociétês de culture pour obtenir les animaux sacrificiels. Ils défrichent les alentours des bois sacrés pour empêcher les feux de brousse, ils réfectionnent les sanctuaires, ils entretiennent les autels, grillent et dépècent les animaux sacrificiels, etc. Les femmes préparent les repas de fêtes et $1 \mathrm{a}$ bière de mil, balaient les places publiques, etc.

Dans le cadre de la communauté familiale, la circulation des produits se fonde sur l'antêriorité des aînés sur les cadets : les seconds sont redevables aux premiers, de la subsistance et des semences. Le même rapport est supposé s'établir entre les aînês et les yapèrè qui procèdent des aînés suprêmes : les ancêtres mythiques. C'est ce rapport d'antériorité qui s'exprime dans les prières adressêes aux ancêtres ou aux yapèrè par les phrases du type : "Voici la bière provenant du mil que tu nous a donné"(9). Le don sacrificiel est possible grâce au mil avancé l'année précédente par la divinité et sa finalité est de perpétuer le cycle : mil-bière sacrificielle-mil. Les aînés manifestent une soumission aux ancêtres et aux yapèrè qui paraissent en position de super-aîné. En réalité, les aînés s'appuient sur l'efficacité (imaginaire) des ancêtres et des yapèrè ; ils ne peuvent fournir aux cadets tout ce qu'ils sont en droit d'attendre d'eux que si les yapèrè et les ancêtres confirment $d$ 'une certaine façon leur prééminence et leur permettent de reproduire les conditions d'existence de la société. Cette fonction (sur laquelle nous reviendrons) justifie de nos jours encore, aux yeux des Minyanka, l'importance de la plupart des rites sacrificiels.

Au niveau matêriel, les yapèrè signifient métonymiquement l'énergie, le courage, l'expérience et toutes les autres qualités des ancêtres. On incorpore en effet aux autels des parcelles du corps des aînés défunts. Quand au cours de la possession ou avant les sacrifices, les maîtres du culte et les chanteurs prononcent les noms de ces ancêtres, ils "réchauffent le nyama" dont sont chargés les yopèrè. A

(9) Autres exemples pp. 97, 98, 100. 
1 'évocation des ancêtres succède celle des vieux initiês qui renouvellent chaque année les racines constitutives des autels. Ceux-ci sont ainsi chargés de force vitale grâce au travail des anciens. Les aînés reconnaissent avec humilité la préêminence des yapèrè et des ancêtres comme en témoigne cette formule classique de la prière :

"Je ne suis qu'un petit enfant, je ne distingue pas mon bras gauche de mon bras droit."

Mais la soumission des aînés aux "dieux" n'est qu'une attitude : elle est d'ailleurs dépourvue de base concrète. A l'inverse de ce qui se passe entre aînés et cadets (les seconds remettant aux premiers l'ensemble des biens produits), les aînés ne cèdent aux "dieux" qu'une petite partie pour recevoir le tout (les conditions naturelles de la production). Cette opération symbolique est diffêrée et imaginaire comme quand on offre de la bière pour obtenir du mil, mais elle peut s'accompagner d'une redistribution réelle comme quand on sacrifie un chien pour la fécondité du village et qu'ensuite sa viande est partagêe.

Les pouvoirs attribuês aux yapèrè masquent un autre rapport social : celui qui place les aînés en position privilégiée par rapport au savoir. Les yapèrè sont aussi la mémoire concrète d'une connaissance des remèdes et des poisons que les aînés ne livrent qu'à leurs conditions aux cadets. I1s contiennent des fragments qui renvoient mêtaphoriquement ou métonymiquement aux substances naturelles qui interviennent dans la pharmacopée. Par la possession des yapèrè et de la connaissance ésotérique et secrète qui s'y rapporte, les aînés ne contrôlent pas seulement des "outils spirituels", ils détiennent une technique véritable. Le pouvoir de tuer ou de guérir est attribué au nya, au nankon, au manyan et à d'autres yapèrè, mais le matériel rituel de tous ces cultes, "gêré" par les aînés, comprend toujours des gourdes ou des paniers pleins de préparations aux vertus thérapeutiques (10). Il ne faut pas négliger non plus le pouvoir psychosomatique d'une pratique médicale enrobée de sacralité.

Les yapèrè se veulent les garants de l'ordre social. Ils répriment les comportements assimilés à des actes de sorcellerie car ils compromettent la perpétuation du groupe lignager : adultère, stérilité, paresse, individualisme, etc.

Comme on le voit la soumission des aînés aux yapèrè, quand bien 106 
même elle serait sincère, cache en réalitê le contrôle qu'ils exercent tant sur le discours idéologique que sur l'usage des remèdes et poisons. Au plus fort de la crise de possession et de l'effervescence religieuse, le médium du nya du village de Wattorosso révèle la vêritable nature de 1 'idéologie religieuse quand il s'écrie :

"Hier ton père et ta mère étaient ton Dieu

tendre les bras vers Dieu, c'est tendre les bras vers tes pères."

B. Le sacrifice dans la société actuelle.

La sociêté minyanka est en pleine évolution. Le changement est perçu comme dangereux car il corrompt les bases de l'organisation lignagère. Ce problème alimente les discussions des aînés. Tel "maître" du nya, assis dans l'enclos sacrificiel de Wolobougou, se plaint de ce que les jeunes ne veulent plus "réparer" (ya) le nya, c'est-à-dire enduire les autels du sang des victimes. Un vieux chef de famille renchérit :

"Les jeunes ne veulent plus travailler pour les chiens, c'est pour cela que la viande manque. Ils ne veulent plus griller les chiens, mais ils ne méprisent pas la viande une fois qu'elle est préparée."

De nombreux cadets s'abstiennent de participer aux rituels. Les jours de cérémonies, ils portent ostensiblement des vêtements européens (alors que cela est expressément prohibé) et les lundi et vendredi, jours normalement consacrés aux sacrifices, ils n'arrêtent pas de travailler.

L'analyse des changements intervenus pendant la période coloniale n'entre pas dans le cadre de cet article. Retenons cependant qu'un certain nombre de facteurs (travail forcé, cultures commerciales, salariat, impôt, pacification, progrès techniques, scolarisation, évangêlisation) ont entraîné la division des lignages. La culture attelée et les engrais rendent aujourd'hui viables de petites exploitations agricolés. Les cadets tendent à s'affranchir des aînés' en ligne collatérale. Ils se détachent de la communauté lignagère mais échappent

(10) Les termes wèrè -- qui signifie à 1'origine "feuilles" -- et yaperè sont d'ailleurs souvent utilisés indifféremment. 
difficilement à son modèle : les nouvelles cellules reproduisent le rapport aîné-cadet au niveau d'ensembles plus restreints. Les chefs de lignage et de segment de lignage voient ainsi émerger des "nouveaux aînés" (chefs de famille étendue) sur lesquels ils parviennent cependant à maintenir une autoritê relative. D'une part, en dépit de $1 \mathrm{a}$ division, les premiers contrôlent et organisent les échanges matrimoniaux. Le mariage par échange d'une soeur classificatoire ne peut en effet se réaliser que dans des groupes comportant suffisamment de filles. D'autre part, les membres du patrilignage (tous les descendants d'un ancêtre commun) continuent à se rassembler à l'occasion des rites sacrificiels autour de leurs chefs traditionnels. Ceux-ci apparaissent toujouts comme les intercesseurs obligés auprès des puissances religieuses. Les cérémonies requièrent la présence des dignitaires des sociétés initiatiques des villages voisins. Une concertation entre aînés (chefs de lignage et de segment de lignage), qui révèle leur solidarité, se déroule à ces occasions. Elle leur permet d'organiser, comme par le passé, les alliances matrimoniales.

L'inscription des sacrifices dans 1 'ordre cosmique contribue jusqu'à présent à justifier la prêéminence des aînés. Aux yeux des Minyanka, les sacrifices aux instances religieuses déterminent les éléments fondamentaux de la production agricole : la terre, la pluie, la fertilité du sol et l'énergie humaine. L'appropriation collective de la terre est projetée dans 1 'imaginaire : "la terre appartient aux ancêtres", et l'appartenance à la communauté lignagère en conditionne 1 'accès. En organisant les alliances matrimoniales, les aînés exercent un contrôle sur la reproduction de l'énergie humaine mais les yapèrè sont censés conditionner la naissance des enfants. La terre travaillée, ensemencée, les agriculteurs estiment nécessaire de se plier aux exigences rituelles des ancêtres et des yapèrè (sacrifices, interdits, etc.) pour qu'ils accordent des pluies régulières et préviennent les catastrophes naturelles (tornades, sauterelles, etc.). Les autels du nya sur lesquels s'écoule le sang sacrificiel témoignent de ces préoccupations comme le révèlent les signes graphiques (11) et les récitṣ initiatiques qui y correspondent. A côtê du signe des ancêtres figurent notamment ceux d'Orion, de Siriuset du calao. Orion pénètre la terre au moment des semailles pour la rendre fertile, Sirius influence la fécondité des femmes et le calao apporte la pluie. Les sacrifices aux "dieux" condi- 
tionnent la santé, la fécondité, la chance et la rêussite de toutes les activitês humaines qui dépendent des ressources naturelles. L'accomplissement scrupuleux des rites aux yapèrè et aux ancêtres doit assurer aux hommes le cours normal de leur existence. L'accès aux instruments de travail (houes, haches, etc.) produits par le forgeron, nous entraîne également dans l'ordre cosmique. Le forgeron, premier homme de la création, héros civilisateur, médiateur, joue dans le déroulement des sacrifices collectifs un rôle de premier plan et nombreux sont les sacrifices individuels à l'enclume. Si aujourd'hui la plupart des forgerons exercent l'agriculture et travaillent le fer moyennant paiement, d'autres continuent, comme par le passé, à dépendre des agriculteurs pour leur subsistance. La quantitē de grains remise au forgeron n'est pas proportionnelle à l'importance de son travail ; il détient, en vertu du statut qui lui attribue la pensée religieuse, un droit au mil, confirmé par les proverbes :

"Un forgeron a droit au mil."

"Un forgeron ne manque jamais de mil."

"Si tu entends qu'un forgeron a faim, c'est que les agriculteurs sont morts."

L'aîné du lignage ou du segment de lignage reste le porte-parole de sa communauté; les femmes et les cadets s'adressent toujours aux "dieux" par leur intermédiaire. Les cultes suscitent aujourd'hui encore un travail supplémentaire que l'on peut mesurer dans certains modes nouveaux (et peut-être transitoires) d'organisation où l'on constate que tous les membres de la communauté lignagère se retrouvent, deux jours par semaine, sur les champs collectifs, dont le produit est affecté spécifiquement aux dépenses religieuses. En outre, nous avons déjà évoqué la "culture des yapèrè" à laquelle se livrent les jeunes pour obtenir les animaux de sacrifice. Enfin, dans les familles où le mode traditionnel de production survit, on prélève dans les greniers collectifs pour faire face aux obligations rituelles.

Les Minyanka lovent dans le sacré les causes efficientes de la production des biens matériels et de la reproduction, mais ils excluent toute possibilité d'appropriation de ces facteurs par un groupe dominant. Les aînés ne constituent pas, dans leur ensemble, une classe sociale. Ils ont été eux-mêmes cadets et leur autorité n'est reconnue

(11) Voir Ph. JESPERS, ibid. 
que dans la mesure aù ils oeuvrent à l'avènement de cadets qui, à leur tour, exerceront l'autorité. Les aînés n'ont qu'un pouvoir de gestion dont la justification s'imbrique dans une philosophie religieuse totalisante.

Malgré 1'influence de 1'économie marchande, l'accès à la terre, la constitution des groupes de travail et les mariages sont toujours déterminés par l'appartenance à la communauté lignagère. La perpétuation des sacrifices cycliques exprime la permanence des principes lignagers.

On observe cependant un glissement dans la nature et la fonction du sacrifice. L'éclatement des lignages constitue une véritable crise sociale au cours de laquelle la religion s'infléchit vers la sorcellerie. Les yapèrè individuels et de sorcellerie prolifèrent. Certains yapèrè sont unilatéralement maléfiques (jini, zantye, ngangan, etc.) ; on les appelle nompsgo ("attacher quelqu'un"). L'opérant noue un fil autour du yapèrè ; il sacrifie, sans invoquer Dieu, en perçant le cou du poulet au lieu de l'égorger. La mort lente qui en résulte est à 1 'image des souffrances infligées à celui dont on prononce le nom à cet instant. Les aînés possesseurs de grands yapèrè collectifs (nya, nankon, manyan) se joignent à une ou plusieurs sociétés de sorcellerie, pour rêffirmer leur pouvoir. Par des paroles et des chants, ils rappe1lent lors des rites sacrificiels 1 'ordre ancien, comme ce possédé du nya qui s'écrie :

"Hier, quand la souris satyene (12) ne suivait pas le chemin de ses pères, elle marchait dans les épines et perdait ses intestins."

Instrument de cohésion des communautés et de respect des chefs de lignage et de segment de lignage, les yapèrè sont devenus aussi le support de l'émancipation de certains cadets. Les yapèrè, qui se transmettait traditionnellement (à l'intêrieur du lignage ou du segment de lignage) par ordre de primogêniture entre frères classificatoires, tendent à s'hériter de père en fils, à moins qu'ils ne soient abandonnés au profit de 1 'islam.

(12) Sorte de souris dont la particularité est de se déplacer en nombre et à la queue leu leu. 